\title{
Driving Style Recognition for Intelligent Vehicle Control and Advanced Driver Assistance: A Survey
}

\author{
Clara Marina Martinez, Mira Heucke, Fei-Yue Wang, Fellow, IEEE, Bo Gao and Dongpu Cao, \\ Member, IEEE
}

\begin{abstract}
Driver driving style plays an important role in vehicle energy management as well as driving safety. Furthermore, it is key for Advance Driver Assistance Systems development, towards increasing levels of vehicle automation. This fact has motivated numerous research and development efforts on driving style identification and classification. This article provides a survey on driving style characterization and recognition revising a variety of algorithms, with particular emphasis on machine learning approaches based on current and future trends. Applications of driving style recognition to intelligent vehicle controls are also briefly discussed, including experts' predictions of future development.
\end{abstract}

Index Terms - Driving style; driving conditions; driver behavior; driving style recognition; machine learning; intelligent vehicle control; energy efficiency; driving safety.

\begin{tabular}{ll}
\multicolumn{1}{c}{ NOMENCLATURE } \\
\hline \hline Acronym & Reference Abbreviation \\
\hline ADAS & Advanced Driver Assisted Systems \\
BEV & Battery Electric Vehicle \\
DS & Driving Style \\
FL & Fuzzy Logic \\
GIS & Geographical Information System \\
GMM & Gaussian Mixture Model \\
GPS & Global Positioning System \\
HEV & Hybrid Electric Vehicle \\
kNN & k-nearest neighbor \\
NN & Neural Network \\
PHEV & Plug-in Hybrid Electric Vehicle \\
RB & Rule-based \\
SVM & Support Vector Machine \\
\hline \hline
\end{tabular}

\section{INTRODUCTION}

C IONNECTED autonomous vehicles and artificial transportation systems require enhanced understanding of the human driver behavior. This is not only necessary to guarantee safe and adequate performance, but also to adjust to

Clara Marina Martinez and Dongpu Cao are with the Centre for Automotive Engineering, Cranfield University, Cranfield, MK43 0AL United Kingdom (e-mails: c.m.marina@cranfield.ac.uk, d.cao@cranfield.ac.uk).

Mira Heucke is with Bosch Gmbh trainee in the junior managers program in Leibniz Universität Hannover.

Fei-Yue Wang is with the State Key Laboratory of Management and Control for Complex Systems, Institute of Automation, Chinese Academy of Sciences, Beijing 100190 CHINA (e-mail: feiyue@ieee.org).

Bo Gao is with AVL Powertrain UK Ltd, Essex, SS15 6SR UK (e-mail: bo.gao@avl.com). the drivers' needs, potentiate their acceptability and ultimately meet drivers' preferences in a safe environment. Therefore, it is essential the recognition of driving style (DS) and driver intention inference for the integration and development of these systems [1] - [7]. This statement is supported by the recently proposed parallel driving framework based on cyberphysical-social systems, where driver behavior and cognition was one of the key elements [8] - [10]. Furthermore, the society is becoming more aware of environmental concerns, which influences decisions made by industry and end users [11] [12]. Particularly, in $200427 \%$ of the global $\mathrm{CO}_{2}$ emissions were caused by the transport sector, being $83 \%$ responsibility of road vehicles [13]. Eco-friendliness, fuel supply security and $\mathrm{CO}_{2}$ regulations are driving research towards more efficient powertrains including: conventional, battery electrical vehicles (BEVs), hybrid electric vehicles (HEVs), plug-in hybrid electric vehicles (PHEVs) between others [12].

Besides, industry has taken a further step to influence driver driving style through active and passive corrective feedback [14] towards safer and eco-friendly practises. The firsts can intervene into the driving task directly by generating haptic inputs [2] [3] [7] [9], whilst the second is only advisory and targets to improve drivers' awareness through visual or audio advice [15] [16]. These systems that provide only feedback to the driver, either haptic or passive, are classified as passive, whilst active systems can interfere directly in the vehicle operation by modifying driver's power demand and recalibrating the components response to compensate for deficient driving habits [13] [16]. Although the feedback suitability and effectiveness can be argued, it has been observed that the simple fact of being monitored already encourages drivers to correct their style [17] [18]. Nevertheless, better understanding of driving style is required to ensure appropriate and consistent recognition and to effectively promote safety and eco-driving.

\section{A. Motivation}

Fuel efficiency is mainly influenced, although not exclusively by: vehicle characteristics, road type, traffic conditions and driving pattern [13] [19-21]. Albeit it is wellknown that DS strongly affects fuel efficiency, there is still limited knowledge of the direct relationship [11]. Furthermore 
fuel consumption could be used to simplify the relationship between DS and vehicle efficiency [12] [22], and aid to characterize drivers [23]. Among the scarce publications that strive towards this direction, Bolovinou et al. developed an algorithm able to calculate the remaining range in a BEV. The authors used route speed and gradient profiles which inherently included information about traffic and DS [24]. Recent publications highlighted a margin of potential benefit of fuel consumption of 20-40\% [15] [25], which agrees with the 5-40\% margin claimed in [26] in simulation environment. However this gain is strongly related to the road type. Mudgal et al. registered 33\% improvement on fuel consumption when correcting aggressive driving on highway, but only $5 \%$ on urban roads [27].

In addition to fuel consumption reduction, road safety has also motivated progress in safety systems. These, according to the degree of automation applied, may also be of intrusive nature: components calibration and vehicle response modification or even pedals and steering control takeover. The complex interaction between these features and drivers has encouraged investigations on human factors involved in car accidents [28]. Some of these factors are: demographics, distraction, experience, fatigue, alcohol, stress, risky behaviour tendency and decision making [29]. Aggressive drivers tend to operate the vehicle in an unsafe manner, including excessive speed, improper car-following, erratic lane changing and imprudent turning manoeuvres [14]. Currently, advanced driver assistance systems (ADAS) are a promising field of research that contributes to safety and powertrain efficiency improvement [30] [31]. Those features assist drivers during specific events [16] [23], but are usually designed based on the average driver characteristics. Although the calibration includes a wide majority, it cannot adapt to specific driver singularities [32]. Hence, the next generation of ADAS features target driver style recognition to individualize the systems performance [30] [31] [33] [34] and potentially improve fuel consumption and safety along with awareness of external driving conditions [21] [35].

Motivated by the previous review, a survey of driving style recognition state-of-the-art and future trends is urged to set the basis for progress in this direction. This paper gathers a review to algorithms and applications of driving style recognition into the current ADAS framework towards increasing levels of automation and vehicle electrification.

\section{B. Driving Style Related Terms}

Driving style is a complex concept influenced by a burdensome number of factors which complicates its description. This has caused the emergence of numerous terms, which usually lack an agreed definition. It is therefore required a concise description to avoid the reader confusion when referencing different authors. The relationships between the terms defined as understood by the authors are illustrated in Fig. 1 to situate the context of driving style and facilitate the comprehension of the terms used in the following.

Driving event is generally understood as manoeuvres occurring during the driving task, such as: acceleration,

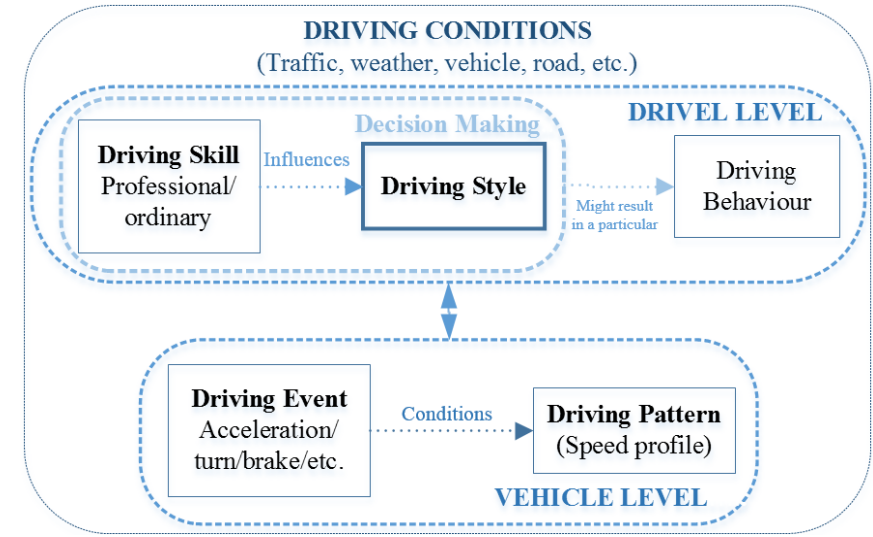

Fig. 1: Driving style-related terminology and connections.

deceleration, turning and lane change, which can be used to identify DS [14]. Henceforth driving pattern is defined in the remaining as the speed profile. This simplified description includes all additional information that can be obtained from the vehicle speed analysis. The previous definition aligns with the literature which designates it as: trip duration and average speed [20], speed profile and calculated power demand [13], both speed and acceleration profile [19], or even consider a detailed scrutiny of the vehicle speed including average number of accelerations and time at constant speed [25].

The driving pattern is strongly related to road type, weather conditions, also referred as driving conditions, and driving style [36], but does not include that information specifically. In contrast, driving behaviour solely focuses on driver' decisions and ignores external factors [20] [25] [26]. These concepts should not be confused with driving skill, which is usually defined as the driver's ability to maintain the vehicle control and is generally used to differentiate between experienced and average ordinary drivers [5].

In contrast to the previous terminology, there is no agreed definition of driver driving style in the literature. This is mainly caused by subjective factors associated to it such as: driver attitude, mood, tiredness, etc. Ishibashi et al. defined it as the attitude and way of thinking towards the driving task [37]. Dörr et al. proposed a pragmatic description, defining it as the way the driving task is accomplished [38]. The concept can be then understood as the manner the driver operates the vehicle: steering wheel, throttle and brake pedal, etc., but with connotations over the driver, as a differentiating factor against driving pattern [38]. Some other definitions concentrate on driver aggression rather than driving style, which is the driver aggressiveness potential. Aggressive drivers usually develop abrupt and unpredictable manoeuvring, which generally results in higher fuel consumption and tends to provoke dangerous situations [28]. Nonetheless, it can be agreed that DS is strongly influenced by the driving external conditions, which cannot be separated from DS evaluation [38] [39]. Hereby driver driving style is understood as the way the driver operates the vehicle controls in the context of the driving scene and external conditions such as time in the day, day of the week, weather and mood, between other factors. This definition agrees with previous descriptions and contemplates 
detecting more than one style for the same driver. That is to say that the same driver could exhibit disparate styles under different conditions, such as: a commuting rote to the work place and a family trip during the weekend.

\section{INPUTS FOR DRIVING STYLE CHARACTERIZATION}

The plurality of definitions of driver driving style is caused by the large number of influencing factors and possible interpretations of the driver response. Some of these variables are listed in Fig. 2. It is unreasonable to expect the control of all factors given the burdensome number and the difficulty to measure most of them. Hereby we differentiate between influencing factors and the actual input signals implemented. These inputs are understood as influencing factors that can be controlled and are chosen to characterize driving style.

\section{A. Driving Style Influencing Factors}

Ericsson conducted a comparative examination of the relationship between driving patterns and external conditions such as traffic, street type and other drivers [19]. The author highlighted the strong influence between these factors and their interactions, necessary to determine DS in the context of the vehicle performance [19]. In contrast, Taubman et al. transferred DS research into a completely different field by analysing human factors: conscious decision making, demographic background and character [28]. The study revealed that new parameters such as driving experience, training and familiarity with the vehicle and the environment can also condition DS. The authors specifically addressed the influence of: gender, age, education and personality by analysing self-esteem, patience, recklessness, anger and extraversion [28]. Likewise, driver state was found to be notably influenced by drugs, fatigue, distraction, etc. in [29].

These previous factors are inner to the driver, but can also be triggered by the external stressing situations such as road state and high congestion level. These, combined with time in the day and day of the week, may affect differently to drivers [19]. The previous analysis opens a debate about DS assignment to each driver. Whilst some researchers allocate a unique style, the previous influences consider the possibility to detect changes in driving style as a response to changes in the external conditions. These results are important from a research perspective, but the implementation of this information for DS recognition might not be feasible. Sociodemographic factors require large amount of data that is not generally available to the recognition algorithm.

A simplified approach for practical DS identification focuses on its influence over single figures, such as: fuel consumption [12] [22], average speed or range. Nevertheless, these indicators might be excessively simplistic to evaluate scenarios with different levels of traffic congestion and fail to represent the complete driving scenario. Besides, the influencing factors may differ depending on the road type. Speed profiles in city routes could become very complex due to turning manoeuvres and multiple braking events, but they could be fully defined by speed and acceleration events in highway roads. The aforementioned highlights the importance
Influencing Factors on Driving Style

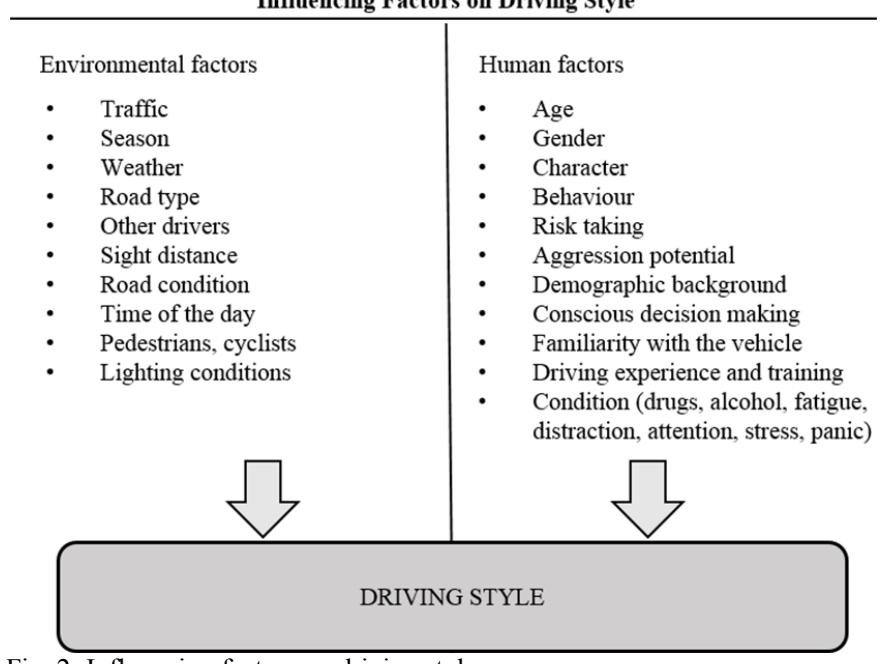

Fig. 2: Influencing factors on driving style.

of road type recognition to ensure correct driving style identification [20]. McCall et al. defined road type and driving conditions recognition as context awareness, information required to properly judge drivers' performance and generate useful feedback. The study was motivated by the fact that drivers classified as "excessively calm" should not be encouraged to drive faster in slippery roads or classified as aggressive in roads where "aggressive" manoeuvres might be required [40]. Murphey et al. suggested more complete road classifications into: freeway, freeway with gradient, arterial and local roads, with incremental congestion level [21]. Xia et al. worked on similar ideas, by proposing fuel economic driving in road segments provided by traffic control infrastructure with vehicle communication of real-time congestion and traffic lights. This approach produced a 10$15 \%$ potential benefit in fuel consumption [41].

\section{B. Role of Sensors}

Although the most suitable factors affecting DS recognition can be argued, they are eventually constraint by the information available. This is limited by the standalone devices that are currently installed in the majority of vehicles in production. A commonly used source of information is the inertial measurement unit [21] [26] [31] [38] [42], which includes: vehicle speed, throttle pedal position, acceleration, etc. Furthermore, low-cost accelerometers can be installed to capture additional information [43]. Vehicle localization can be provided by the Global Position System (GPS), which is simultaneously an indirect measurement of speed and acceleration [17] [27]. More advanced vehicles include devices to detect the driver behaviour under specific manoeuvres, such as Geographical Information System (GIS) used for location of roundabouts [27]. In addition, the use of parameters related to car-following would require the installation of radar or LiDAR sensors, already available in vehicles featured with adaptive cruise control [34] [38] [44]. When the desired signals are not available, smartphones can be used instead [13] [14] [16] [18]. Due to the increasing popularity of these devices, it is reasonable to assume their 
presence and availability. Smartphones can contain: accelerometers, gyroscopes and geomagnetic field sensors [18], GPS and cameras [14].

Table I summarizes the vehicle sensors introduced. These approaches for signals capturing and sensor installation respond to discrepancies in terms of electronics installed in vehicles currently produced. Given the tendency of further electrification, it can be presumed that future vehicles will be provided of an integrated sensor system able to provide all required information.

\section{Driving Style Recognition Input Signals}

The initial step for driving style identification consists of determining the variables necessary to monitor so as to provide a robust classification. Choosing the correct signals is crucial since any further processing and results depend entirely on it. Nevertheless, there is no general agreement of a recommended set of parameters in the literature [19] [28]. This disagreement responds to the plurality of applications of DS for: driver correction, fuel consumption reduction, safety enhancement, etc. A summary of the solutions proposed by the reviewed authors is included in Table II.

Ericsson explored a holistic study of independent measures on DS with respect to emissions and fuel consumption. The initial set of 62 signals is simplified by discarding unnecessary information into 26 including: speed mean and standard deviation, acceleration, deceleration, speed jerkiness in curves and proportion of time at different speed. This set is further reduced to 16 independent factors, where only 9 where directly relevant to fuel consumption. The analysis highlighted the importance of determining road type and traffic conditions prior to DS recognition, due to the strong relation between the parameters and the level of congestion [20].

Although the previous author performed a significant reduction of the initial set of parameters, DS algorithms implementable in-vehicle real applications require further simplifications to comply with cost and running time limits. More simplistic approaches take advantage of the relationship between aggressive drivers and high acceleration [18], speed profiles and fuel/energy/power consumption [13] [26] [33]. Acceleration is generally complemented with other variables such as braking or velocity statistics [18] [45] [46], being the most common combination the measures of speed, acceleration and deceleration or similar. In contrast, other variables such as jerk [21], braking pressure and usage of the throttle and brake pedals were also widely employed and even understood as better indicators by many authors [21] [23]. The combination of both longitudinal and lateral dynamics has been also highlighted for being able to capture the majority of the vehicle motions [34]. Nevertheless, these approaches only provided complete information when the driving situation was also known, named weather conditions, traffic and road type [21]. Furthermore, although DS is apparent for conventional and $\mathrm{BEV}$, it is particularly critical for HEV and PHEV due to the combination of at least two power sources [47]. Small changes over the torque request can trigger the transition into different hybrid modes and affect the amount of energy
TABLE I

COMMON SENSORs REQUiRE FOR DRIVING StYle Signals CAPTURING

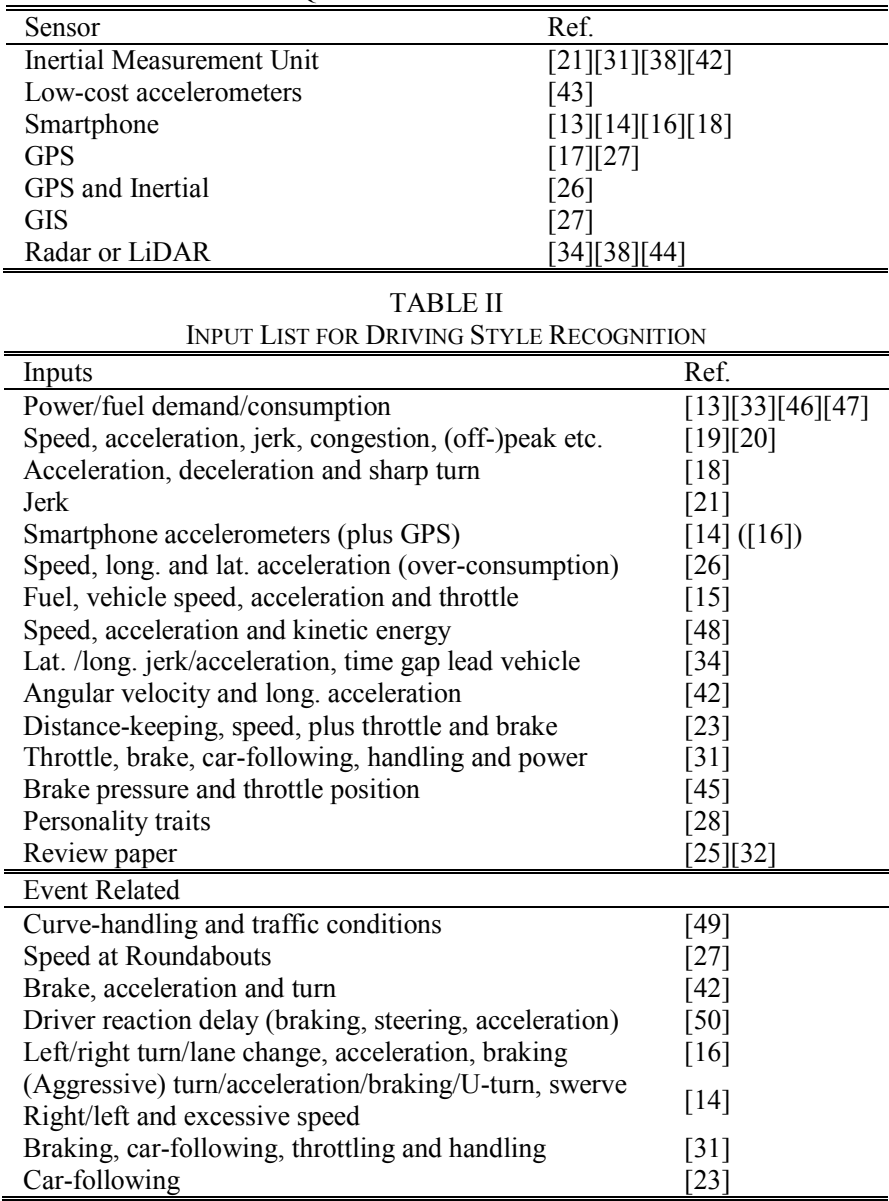

recovered during braking [48]. These studies target fuel oriented identification implement power, energy or fuel consumption measures [48].

Signals selection can be guided by the identification of specific events [14], which are tightly related to the vehicle dynamics and contain information about the driver intention. The key driving events identified are usually extracted from the data [42] and include: braking, car-following, distance keeping [23] [32], roundabouts [27], handling, including lane change and cornering [16] [31] [49] and the reaction delay to events [50]. This information can be combined with additional signals and statistics to enhance the classification [31]. The former encompasses different interpretation of speed and acceleration profiles according to events or even adjusting the signal set to specific manoeuvres.

\section{DRIVING StYle ClassificAtion}

Signal selection for DS is strongly linked to the classification criteria and recognition algorithms used as illustrated in Fig. 3. Due to the large number of signal candidates and applications, there are numerous approaches for DS classification with different labelling. A survey of the main criteria and classification options included in the following is summarized in Table III. 


\section{A. Driving Style Classification into Discrete Classes}

The general tendency is to group driving styles into classes based on the distribution of the selected driving parameters and extracted features [25]. These classes need to be defined prior to the classification algorithm design and should account for all influencing parameters in a multifactor cognition task. They need to account for the trade-off between classification finesse and complexity to guarantee the algorithm robustness and have to be understandable by the end user [34]. Classes labelling can be inspired from either safety or fuel economy perspectives. Whilst safety related applications refer to the level of aggressiveness, fuel oriented classification generally uses terminology related to efficiency or sportiness. The plurality of classes labelling further increases when combining the previous with increasing number of DS groups.

The revision to the literature reveals a prevalence of simple classification bases using either two or three categories. This solution provides easily understandable feedback and facilitates the algorithm calibration. The distinction between aggressive and nonaggressive was use in [14] [16] [30] [34] [43], fuel economic vs. performance [15]. Aggressive drivers often show careless handling behaviour and may result in higher fuel consumption [45]. They exhibit frequent changes in the throttle pedal position of large magnitude, whilst calm drivers only show small amplitudes and low frequency. The division into three classes includes aggressive, mild and moderate styles, as defined by Xu et al. [45], soft, normal and aggressive by Syed et al. [15] or comfortable, normal and sporty as described by Dörr et al. [38]. Moderate drivers are described as an intermediate group that shows properties from both aggressive and calm, but without conclusive membership to any of those.

In contrast to the previous, Taubman et al. presented a study on multidimensional driving style which examined human factors involved in car accidents and inspired a safety related classification: reckless and careless driving, anxious driving, angry and hostile driving and patient and careful driving [28]. Other authors opted for a more precise classification using higher amount of grades by identifying the no speed category [21], whilst Constantinescu et al. proposed a five to seven classes, covering the range from non-aggressive to aggressive style [17]. Larger number of clusters improves the classification finesse, but complicates the algorithm development and the interpretation of the classes themselves. DS identification is of no use unless the information provided is clear, statement supported by Table III that situates the recommended number of cluster below 5 .

\section{B. Driving Style Classification through continuous Indexing}

The ultimate classification case into a larger number of clusters consists of considering a continuous index, which eventually could be used in a threshold-based algorithm to transform it into finite classes [21] [51]. Augustynowicz classify DS in a range within $(-1,1)$, being $-1,0$ and 1 mild, normal and aggressive respectively [51]. This index is generally calculated from the relative fuel consumption or overall efficiency, instead of driver level of aggressiveness.

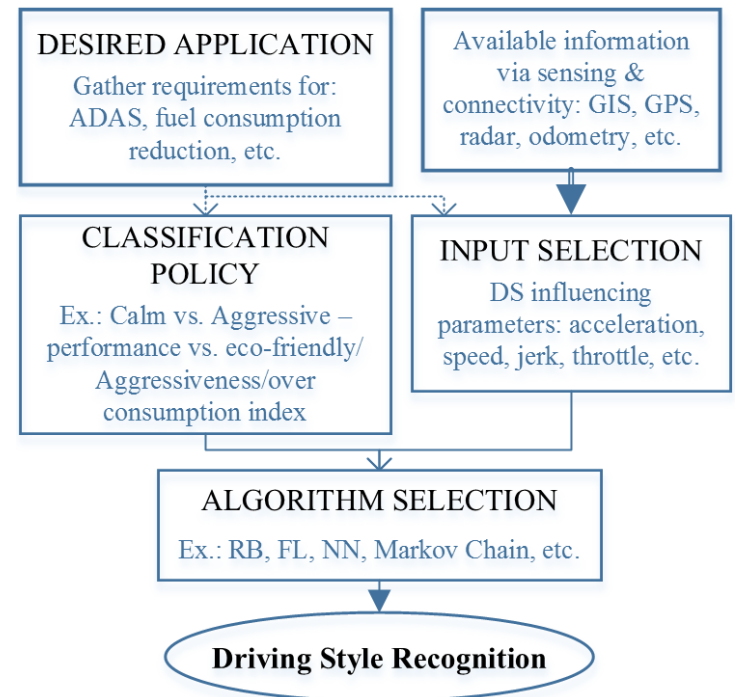

Fig. 3: Driving Style Recognition Algorithm Design Process.

TABLE III

IDENTIFICATION BASIS AND CLASSIFICATION DETAILS FOR DRIVING STYLE

\begin{tabular}{|c|c|c|}
\hline Ref. & $\begin{array}{l}\text { No. Class } \\
\text { or Rank }\end{array}$ & Classification Details \\
\hline $\begin{array}{l}{[11][12][14][15]} \\
{[16][30][34][43]}\end{array}$ & 2 & Aggressive/calm (unsafe/safe) \\
\hline$[15][19][25][38][45]$ & 3 & Aggressive/moderate/calm \\
\hline [21] & $3+1$ & $\begin{array}{l}\text { Aggressive/moderate/calm + no } \\
\text { speed }\end{array}$ \\
\hline [18][39] & 4 & Very bad / bad / good / very good \\
\hline [17] & $5-7$ & Calm - aggressive / steady - dynamic \\
\hline$[13][20][26][48]$ & Rank & (Over) fuel / power consumption \\
\hline [28] & & Reckless/anxious/angry/patient \\
\hline [51] & $\operatorname{Rank}(-1,1)$ & From most-aggressive to most-mild \\
\hline
\end{tabular}

Manzoni et al. used an estimation of the fuel consumed along the trip and compared it to a benchmarked value to calculate an over-consumption percentage indicative of extra cost [26]. Fuel consumption was also used by Neubauer et al. to obtain the vehicle efficiency as indicative of DS [48]. Similarly Corti et al. assessed driver driving style with an energy-oriented cost function that estimated the power over-consumption [13].

\section{DRIVIng Style Recognition Algorithms}

Driving style recognition algorithms are developed on the basis of the selected classification method and signals employed. DS recognition algorithms are usually implemented through a set of rules, via models or using machine learning as summarized in Fig. 4. Although implemented through a specific strategy, these algorithms can be developed combining some of the previous. For instance, rules can be inspired on data processed through unsupervised machine learning. Table IV presents a concise summary of the main algorithms reviewed in this section and respective references.

\section{A. Implemented through Rules}

Rule-based (RB), also referred as threshold-based algorithms, are the simplest approach for DS recognition. Rules are defined based on prefixed thresholds over the monitored variables that allocate DS into groups. These rules are usually defined for specific events, as introduced by 
Radoslav who implemented a RB algorithm particularized to six driving events: acceleration, deceleration, left/right turn and left/right lane change. DS was obtained through a final score that counts the relative number of aggressive manoeuvres [18]. Murphey et $a l$. presented a similar approach based on aggressive manoeuvres counting. The final score was obtained in percentage basis, distinguishing within: calm below $50 \%$, aggressive above $100 \%$ and normal otherwise [21]. Alternatively to driving events classification, DS can be grouped according to fuel or overall energy consumption as already included [13] [26]. The use of RB algorithms unifies simplicity, easy interpretation and implementation, but limits the amount of parameters that can be managed. The previous examples are generally based on a single parameter, and therefore the robustness and accuracy of the results are considerably limited.

Larger sets of variables generate rules unnecessarily complex that can be replaced by fuzzy logic (FL) maps. These are also based on prefixed thresholds but are able to include more parameters whilst keeping its simplicity, robustness, easy understanding and low computational order. Dörr et $a l$. developed a real-time algorithm that also considered other variables such as road type and gap between vehicles by implementing FL. This system was verified in simulation environment in urban and rural road without traffic disturbances achieving $68 \%$ of correct classification rate and $2 \%$ of incorrect classification rate [38]. Gilman et al. also employed a RB and FL based on a total of 17 factors evaluated through a performance indicator [39]. Syed et al. proposed a FL algorithm to evaluate optimal pedals operation in a HEV. This algorithm monitored both throttle and braking pedals operation and was able to calculate an appropriate correction and generate haptic feedback to the driver. The authors claimed a minimum of $3.5 \%$ improvement in fuel consumption for mildest driving in simulation environment, without compromising the vehicle performance [47]. Won also used FL to improve HEV fuel consumption using road type and events detection: start-up, acceleration, cruise, deceleration and stationary [52]. A similar approach was taken by Kim et al., who used a FL control with driving mode, driving style and driving conditions recognition capability. This was utilized to adapt the control of a HEV and BEV given pedals operation and external temperature and tune the state of charge window, battery recharge/depletion and engine on/off state thresholds. The state of charge membership functions were hereby adaptive to the driving conditions [53].

Although RB and FL algorithms unify acceptable results with implementation simplicity, the quality of the classification is strongly related with the selection of the thresholds. These can be based on the expertise of the designer, although more strictly should be inspired on real data. Maximum number of drivers should be involved in the data collection to gather maximum data variability and generalizing capacity. These algorithms are also limited in terms of the amount of variables and data that can process, reason why they might be compensated with data-driven algorithms at least at the development stage. The next

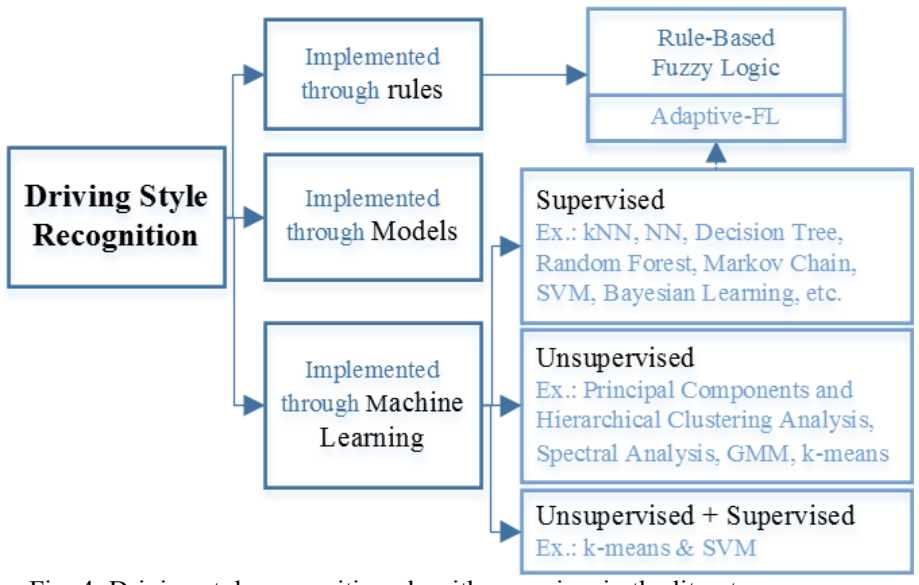

Fig. 4: Driving style recognition algorithms review in the literature.

TABLE IV

AlgORITHM AND DATA SOURCES/INPUTS FOR DRIVING STYLE RECOGNITION

\begin{tabular}{ll}
\hline \hline Reference(s) & Algorithm \\
\hline$[11][13][18][21][26][28]$ & RB \\
{$[17][37]$} & RB (Clustering Analysis) \\
{$[31][38][47][52][53]$} & FL \\
{$[39]$} & RB and FL \\
{$[15]$} & Adaptive- FL \\
{$[45]$} & NN \\
{$[51]$} & Elman-NN \\
{$[30]$} & kNN, NN, Decision Tree, Random Forest \\
{$[14]$} & kNN Dyn. time wrapping \\
{$[43]$} & kNN \\
{$[27]$} & Hierarchical Bayesian Regr. \\
{$[40]$} & Bayes Learning \\
{$[42]$} & K-means and SVM \\
{$[55][56]$} & Markov Chain \\
{$[35]$} & GMM, k-means, SVM, etc. \\
{$[23][25]$} & GMM \\
{$[33]$} & Monte Carlo Markov Model \\
{$[32]$} & Review Paper \\
\hline \hline
\end{tabular}

generation of algorithms require the capability to account for larger data sets and influencing factors, characteristics that escape from RB limits and point to their combination with machine learning approaches, between others.

\section{B. Implemented through Models}

Model-based algorithms consist of the description of driving style through a set of equations of pre-defined characteristics. The models selected are adjusted to each DS by tuning their parameters to fit the data used to inspire them, usually using data-driven methods. The models complexity is given by the application requirements and influences the fidelity of driving style representation. Driving style models respond to the necessity to reduce data collection and testing, which is time consuming and involves high costs. Driver models can be used to reproduce DS in simulation environment, test ADAS features in more realistic environments [38] and adapt ADAS performance in real-life applications (driver-in-the-loop). Furthermore, advanced driver models can replace classification algorithms allocating real world driving scenarios simulated into previously established classes. Nevertheless, driver modelling has as main drawback in the difficulty to prove the accuracy of the results. Model validation requires their comparison with real drivers, which again necessitates extensive data collection [32]. 


\section{Implemented through Learning Algorithms}

Threshold definition in RB algorithms conditions the results robustness and requires extensive data analysis. Data-driven algorithms are suitable to process large amount of data and consistently derived the thresholds value or even fully develop improved algorithms. Furthermore, these have adaptive capability that can be applied to particularize to specific drivers. These beneficial characteristics have promoted research into data-driven approaches and is conditioning future trend in DS recognition. Proof of that is found in the extensive literature available and represented in the following with a predominant coverage with respect to RB and modelbased approaches.

\section{1) Unsupervised Machine Learning Algorithms}

Unsupervised algorithms do not require understanding of the underlying process. The classification is achieved through statistical analysis of the input signals inherent to the algorithms. This allows increasing the number of classes and therefore the finesse of the classification. Constantinescu et al. proposed two alternative algorithms based on hierarchical cluster analysis and principal components analysis. The first one identifies groups of drivers based on similarities in the driving statistics, whilst the second option studies the correlation between variables to transform the original data set into a smaller one, whilst keeping the useful information. Each algorithm returned a different number of classes, 5 and 7 in principal components and hierarchical clustering respectively [17]. The use of principal components with extensive analysis of the driving-related extracted features is included in [37].

Miyajima et al. implemented a Gaussian Mixture Model (GMM) based on car-following behaviour and pedals operation spectral analysis. The model achieved an identification rate of $69 \%$ car-following, whilst the pedal spectral analysis provided a classification rate of $89.6 \%$ in simulation environment and $76.8 \%$ in field tests [23]. A similar application of GMM for pedal operation modelling was also reviewed by Wang and Lukic in [25]. Johnson and Trivedi applied dynamic time wrapping to detect aggressive events with a $97 \%$ success in simulation environment [14].

Driving style statistical characteristics also encourages the use of Bayesian methods. Mudgal et al. implemented a hierarchical Bayesian regression model to characterize DS in roundabouts [27]. Similarly, McCall and Trivedi employed Bayesian learning to assess critical situations related to braking assistance. The model was used to assess when a braking would be required and the probability of the driver performing this manoeuvre [40].

Unsupervised algorithms have proved their applicability and suitability for DS recognition. Nevertheless, the output needs to be directed in terms of interpretation and number of clusters. Besides, the classification performance can be worse compared to supervised algorithms.

\section{2) Supervised Machine Learning Algorithms}

Supervised algorithms imply knowledge of the driving style of the data used for training. One of the simplest supervised algorithms is k-nearest neighbour (kNN), which is based on similarity measures rated by the "votes" of the predefined kneighbours. This method was exploited by Vaitkus et al. using a total of five features extracted from the data, which provided a $100 \%$ classification success using experimental data from 110 routes. Furthermore, the authors claimed $98 \%$ classification success using only 3 features based on central statistics. Nevertheless, these results needed to be evaluated based on the characteristics of the training and testing data which proceeded from the same route, season and analogous traffic conditions [43].

An Elman-type neural network (NN) was applied by Augustynowicz to identify DS using speed and throttle pedal position [51]. Another application of $\mathrm{NN}$ was proposed by $\mathrm{Xu}$ et $a l$. who developed a distal learning control framework with two feedforward networks. NN1 concerned the plant inverse model and NN2 simulated the plant forward model, which was run in parallel for error detection and self-learning [45]. A comparison between various supervised learning algorithms was performed by Karginova et al. who implemented: kNN, $\mathrm{NN}$, decision tree and random forest. DS recognition using $\mathrm{kNN}$ achieved its best performance within nearest neighbour inherent limits when clustering for $\mathrm{k}=4$ or 5 . NN optimal number of hidden nodes was obtained as 20 with two approaches. The first network was trained with unmodified data, whilst the second was trained twice with normal training data to encourage the preference for this style. As expected, the second NN showed less incorrect classification of normal DS. Random forest implementation was performed to improve the poor performance of a single decision tree. The tests revealed an optimal number of 40 trees, which achieved the best results in simulation environment. Again the algorithm performance in normal style classification was high, but returned unsatisfactory results in aggressive style classification. The algorithms assessment showed applicability in real-time classification in all cases, but preference for $\mathrm{NN}$ candidates based on the classification performance [30].

Markov models have also been successfully implemented for DS recognition. In Markov chains, future states only depend on the current states, whose transition is generally described by the so-called transition probability matrix [54]. The stochastic and random characteristics of driver behaviour can be captured using Markov models as claimed by Guardiola et $a l$., who defended the benefits of Markov Models combined with Monte Carlo application to generate random patterns based on past data in representation of DS [33]. Pentland and Liu defended Dynamical Markov Model suitability to capture DS, supported by the fact that human actions are best captured as a sequence of control steps [55]. Similarly, a Hidden Markov Model was used by Nechyba and $\mathrm{Xu}$ to learn human control and validate the results [56].

3) Combined Unsupervised and Supervised Algorithms

Although supervised and unsupervised learning could be interpreted as alternative methods, these can be combined in two-part algorithms to benefit from their advantages and improve the overall performance. Unsupervised learning contributes to identify the relevant input signals and the most efficient classification strategy, whilst supervised learning generally returns better classification results. Bolovinou et al. provided a survey of data-driven algorithms combining supervised and unsupervised methods based on Bayesian inference, GMM, Support Vector Machine (SVM), K-mean clustering, Bayesian Network and Markov models. However no concluding comparison was delivered due to the 
incompatibility caused by differences in the input data and DS definition [35]. Van et al. explored the application of K-means along with SVM. K-means unsupervised algorithm was used to explore the relevance of specific manoeuvres and their combination along with the driver pedals operation, whilst SVM was used for implementation [42].

\section{INTELLIGENT VEHICLES AND CONTROL APPLICATIONS}

DS recognition has several applications such as: driving style feedback and correction, driver-in-the-loop, ADASs performance enhancement and energy management in $\mathrm{HEV}$, PHEV and BEV. These can be gathered in two main groups, safety or fuel efficiency aware. These alternative classifications can correlate at times given the fact that aggressive driving is generally associated to inefficiency, but do not coincide in all cases. That is, calm drivers do not necessarily drive efficiently.

\section{A. Driver Advisory Feedback}

One of the main applications of DS recognition targets driver style correction through either online or offline feedback, which can be passive, active or a combination of both. Corrective systems can be directly installed in the vehicle or included in smartphones to make use of the device sensors and processing capability [13] [14] [18]. An example of DS recognition and correction using smartphones was presented by Stoichkov. The application was designed to display visual feedback to promote DS improvement for both fuel consumption and risks of accidents reduction [18]. An alternative approach was proposed by Fazeen et al. who generated instead audio feedback to encourage DS correction [11], as is similarly used in [14]. Corti et al. designed an algorithm with three alternative levels of visual information tested against the no-feedback scenario. All approaches showed improvement in the vehicle consumption up to 20$30 \%$, although best results were achieved when providing enough information in a clearest and most understandable manner [13]. Doshi and Trivedi went a step further and analysed the response of drivers to the feedback to adapt the information facilitated and obtain individual evaluation [34].

Although passive feedback strategies are easy to implement and can return satisfactory results, it always exists the possibility that the driver chooses to ignore the guidance. In contrast, active feedback systems generate a response that affects the normal vehicle operation, which cannot be as easily neglected. Syed et al. provided both visual and haptic feedback registering up to $3.5 \%$ improvement in fuel economy without reducing the expectations of vehicle performance as claimed by the authors. Haptic feedback was generated through the throttle pedal [45] [57]. Nevertheless, haptic guidance can easily cause the driver annoyance, which could lead to the system manual deactivation. This issue was palliated by Syed et al. in [15] and [58], who analysed drivers' response acceptance to the feedback and adjusted it to reduce its intrusiveness to less receptive users. Reichart et al. described a similar approach, by increasing gradually the resistance over the throttle pedal when the vehicle operation diverges from optimal use adaptive to the driver response to the feedback [59].

Alternative approaches are especially designed to improve safety and efficiency in larger vehicles such as busses and fleet management [17] [26] [43] and even passengers comfort in public transport [17]. Drivers of these means of transport could benefit from a personalized driving assistance, "Driving coach", as described by Gilma et al., system provided by context awareness to adapt to specific routes [39]. Rossetti et al. proposed the use of serious game in transportation as educational technique to improve driving skills in simulators by analysing behaviour assimilation, elicitation and persuasion [60]. Future trends of driver feedback will probably lean to a personalized assistance, where the system will be able to detect the driver preferences and generate consistent feedback to both encourage style correction and avoid driver annoyance. Ideally, this guidance should combine haptic and passive feedback with appropriate amount of information easily to interpret and follow, based on the driver cognitive capacity and skills.

\section{B. Enhance of ADAS Performance}

As previously mentioned, safety related systems would benefit from knowledge about DS to predict and anticipate to drivers' reactions and adjust to individual users. ADASs adaptation to drivers may result in safety systems of better performance [25] [34]. This would be the case of collision avoidance systems that include driver-in-the-loop, which would be able to anticipate to drivers' reactions and actuate accordingly [9] [40]. Nonetheless, implementing ADASs in driver-adaptive manner is a complex task that requires further investigation in the appropriate way of using DS information in intelligent vehicles [35]. Kurz et al. presented a method to classify and use DS to improve the vehicle handling based on an online/offline algorithm updated with historical data. The authors also propose person-specific implementation for multiple drivers [50].

Additional safety-related applications can be found in cooperative driving frameworks such as tandem and platoon, as exposed by Rass et al. The system assumed the drivers were willing to cooperate and provided feedback to encourage safety and improve the overall fleet efficiency [61]. Similar ideas were reviewed by Fotouhi et al. to reduce fuel consumption and emissions [62]. Safe autonomous driving can be an ultimate application of DS throughout increasing levels of automation. Urmson et al. proposed an algorithm able to learn from drivers during manual operation and reproduce human-like manoeuvres autonomously within pre-established safety constraints [63].

\section{Control of HEV, PHEV and BEV}

Although conventional vehicles are strongly affected by DS, hybrid and battery electric vehicles are less sensitive to it due to the higher efficiency of the electric components, idling reduction and regenerative braking. Lenaers [46] asserted the influence of DS and road type combinations on consumption and $\mathrm{CO}_{2}$ emissions in four different powertrains: petrol, diesel, 
hybrid and liquefied petroleum gas. The hybrid powertrain was able to provide lower consumption even under urban aggressive drive, particularly due to start-stop and regeneration during braking. Furthermore, aggressive driving styles was found to require up to $68 \%$ and $47 \%$ more fuel in urban and rural roads respectively in conventional powertrains, whilst liquid petroleum and most importantly hybrid powertrains are less sensitive to the different driving patterns [46]. Neubauer et al. compared the effect of DS on a conventional vehicle, HEV, PHEV and BEV in terms of optimal operating conditions. The results showed important influence of driving style in all vehicle platforms, although this was affecting differently. HEV presented optimal working points at low speed, whilst conventional vehicles improved the overall efficiency at higher speed values. Furthermore, HEVs were barely affected by braking due to the regenerative capability, but particularly vulnerable to high acceleration [48]. A part from different operating conditions, hybrid modes selection in HEV and PHEV are strongly related with the driver torque request and inevitably to DS. Consequently, slight changes in driving style can cause unnecessary modes switching and develop sub-optimal performance [15].

Besides, energy management can be enhanced with prior knowledge of driving style in HEV and PHEV. The increasing level of vehicle electrification has motivated research on the effect of DS and driving events on the energy demand in PHEVs, being both contributions particularly important for optimal control [12]. An optimal control strategy can be obtained with knowledge of the entire driving cycle, environment and driver behaviour. Thus the information about driving style can potentially lead to important fuel savings [25]. Driving style information was used by Lin et al. to improve the energy management of a hybrid truck [64]. Yu et $a l$. used driving pattern and style recognition to better estimate the remaining range in PHEV assuming static and quasi-static knowledge of the route [65]. Similarly, Zhang et al. proposed an adaptive control strategy for a PHEV using driving pattern recognition [66].

Driving style recognition can also provide useful information to estimate more accurately the remaining range, improve range management and potentially extend it [22]. The effect of DS over the BEV range was highlighted by Bingham et $a l$., who claimed a possible divergence of $10 \%$ between drivers. This margin was associated to aggressive deceleration events that triggered friction brakes [67]. Similarly, Bolovinou et $a l$. developed a strategy able to estimate the remaining range using route information: speed, gradient profile, traffic conditions and DS. This study targeted an accurate estimation of the battery range to reduce the so-called range anxiety that characterises BEV drivers and is found to be the main constraint that hinders electric vehicles integration in the market [24].

\section{CONCLUDING REMARKS}

This article offers a review of the recent research and development efforts on driving style characterization and recognition as well as their applications to intelligent vehicle control. The design process is chronologically followed from input signals identification and classification policy definition to the algorithm selection and implementation. All driving style influencing factors and classification strategies are presented in relationship to the targeted applications and implementation constraints. The complexity of driving style is discussed and the predominant interpretations, safety and fuel efficiency-related, are analysed through the enumeration of different algorithms. The gradual increment of ADAS presence and vehicle autonomous capabilities requires deeper driving style analysis and the inclusion of drivers in the systems. This has promoted the use of data-driven algorithms able to process more data and the implementation of machine learning algorithms able to adapt to individual drivers. Future trends will focus on even larger data sets covering a broader plurality of drivers and will implement the combination of supervised and unsupervised algorithms to enhance driver adaptability and cognitive performance. The presented review positions driving style recognition as a crucial concept for intelligent vehicle development that will strongly condition the progress in autonomous vehicles and their integration in the market.

\section{REFERENCES}

[1] F.-Y. Wang, (2010), Parallel control and management for intelligent transportation systems: Concepts, architectures, and applications, IEEE Transactions on Intelligent Transportation Systems, Vol. 11, No. 3, pp. 630-638.

[2] L. Li, D. Wen, N. N. Zheng and L. C. Shen, (2012), Cognitive cars: A new frontier for ADAS research, IEEE Transactions on Intelligent Transportation Systems, Vol. 13, No. 1, pp. 395-407.

[3] J. Zhang, F.-Y. Wang, K. Wang, W. H. Lin, X. Xu and C. Chen, (2011), Data-driven intelligent transportation systems: A survey, IEEE Transactions on Intelligent Transportation Systems, Vol. 12, No. 3, pp. 1624-1639.

[4] Dong, Y., Hu, Z., Uchimura, K., and Murayama, N., (2011), Driver inattention monitoring system for intelligent vehicles: a review, IEEE Transactions on Intelligent Transportation Systems, Vol. 12, No. 2, pp. 596-614.

[5] T. Qu, H. Chen, D. Cao, H. Guo and B. Gao, (2015) Switching-based stochastic model predictive control approach for modelling driver steering skill, IEEE Transactions on Intelligent Transportation Systems, Vol. 16, No. 1, pp. 365-375.

[6] Y. Zheng, S. Li, J. Wang, D. Cao and K. Li, (2016), Stability and scalability of homogeneous vehicular platoon: study on influence of information flow topologies, IEEE Transactions on Intelligent Transportation Systems, Vol. 17, No. 1, pp. 14-26.

[7] K. Bengler, K. Dietmayer, B. Farber, M. Maurer, C. Stiller and H. Winner, (October 2014), Three decades of driver assistance systems: review and future perspectives, IEEE Intelligent Transportation Systems Magazine, Vol. 6, No. 4, pp. 6-22.

[8] F.-Y. Wang, (2005), Agent-based control strategies for smart and safe vehicles, IEEE International Conference on Vehicular Electronics and Safety, pp. 331-332.

[9] F.-Y. Wang, (2014), Scanning the issue and beyond: parallel driving with software vehicular robots for safety and smartness, IEEE Transactions on Intelligent Transportation Systems, Vol. 15, No. 4, pp. 1381-1387.

[10]Barfield, W., and Dingus, T. A., (2014), Human factors in intelligent transportation systems, Psychology Press, Virginia Polytechnic Institute and State University.

[11]T. Lee and J. Son, (2011), Relationships between driving style and fuel consumption in highway driving, SAE Technical Paper, No. 2011-280051.

[12]Padmarajan, B.V., McGordon, A. and Jennings, P. A., (May, 2012), An investigation on the effect of driver style and driving events on energy demand of a PHEV, Electric Vehicle Symposium 26, Los Angeles CA, pp. $1-9$. 
[13]Corti, A., Ongini, C., Tanelli, M., and Savaresi, S. M., (2013), Quantitative driving style estimation for energy-oriented applications in road vehicles, IEEE International Conference on Systems, Man, and Cybernetics, pp. 3710-3715.

[14]D. Johnson and M. Trivedi, (2010), Driving style recognition using a smartphone as a sensor platform, IEEE Conference on Intelligent Transportation Systems, pp. 1609-1615.

[15]F. Syed, S. Nallpa, A. Dobryden, C. Grand, R. McGee and D. Filev, (2010), Design and analysis of an adaptive real-time advisory system for improving real world fuel economy in a hybrid electric vehicle, SAE Technical Paper, No. 2010-01-0835.

[16]M. Fazeen, B. Gozick, R. Dantu, M. Bhukhiya and M. Gonzalez, (2012), Safe driving using mobile phones, IEEE Transactions on Intelligent Transportation Systems, Vol. 13, No. 3, pp. 1462-1468.

[17]Z. Constantinescu, C. Marinoiu and M. Vladoiu, (2010), Driving style analysis using data mining techniques, International Journal of Computers, Communications \& Control, Vol. 5, No. 5, pp. 654-663.

[18]R. Stoichkov, (2013), "Android smartphone application for driving style recognition," Project Thesis, Technische Universität München, Munich, advisor: Kranz, M. and Diewald, S.

[19]E. Ericsson, (2000), Variability in urban driving patterns, Transportation Research Part D: Transport and Environment, Vol. 5, No. 5, pp. 337-354.

[20]E. Ericsson, (2001), Independent driving pattern factors and their influence on fuel-use and exhaust emission factors, Transportation Research Part D: Transport and Environment, Vol. 6, No. 5, pp. 325-345.

[21]Y. L. Murphey, R. Milton and L. Kiliaris, (2009), Driver's style classification using jerk analysis, Computational Intelligence in Vehicles and Vehicular Systems, pp. 23-28.

[22] J. Neubauer and E. Wood, (2014), Thru-life impacts of driver aggression, climate, cabin thermal management, and battery thermal management on battery electric vehicle utility, Journal of Power Sources Vol. 259, pp. 262-275.

[23]C. Miyajima, Y. Nishiwaki, K. Ozawa, T. Wakita, K. Itou, K. Takeda and F. Itakura, (2010), Driver modeling based on driving behavior and its evaluation in driver identification, Proceedings of the IEEE, Vol. 95, No. 2, pp. 427-437.

[24]Bolovinou, A., Bakas, I., Amditis, A., Mastrandrea, F. and Vinciotti, W., (2014), Online prediction of an electric vehicle remaining range based on regression analysis, IEEE International in Electric Vehicle Conference, pp. $1-8$.

[25]R. Wang and S. Lukic, (2011), Review of driving conditions prediction and driving style recognition based control algorithms for hybrid electric vehicles, Vehicle Power and Propulsion Converence, pp. 1-7.

[26] V. Manzoni, A. Corti, P. De Luca and S. Savaresi, (2010), Driving style estimation via inertial measurements, IEEE Conference in Intelligent Transportation Systems, pp. 777-782.

[27] Mudgal, S. Hallmark, A. Carriquiry and K. Gkritza, (2014), Driving behavior at a roundabout: A hierarchical Bayesian regression analysis, Transportation research part D, pp. 20-26.

[28] O. Taubman-Ben-Ari, M. Mikulincer and O. Gillath, (2004), The multidimensional driving style inventory - scale construct and validation, Accident Analysis and Prevention Vol. 36, No. 3, pp. 323-332.

[29] "www.exponent.com," Exponent Engineering \& Scientific Consulting, 2010. Available: http://www.exponent.com/human_factors_vehicle/. [Accessed 16 May 2015].

[30]N. Karginova, S. Byttner and M. Svensson, (2012), Data-driven methods for classification of driving styles in buses, SAE Technical Paper, No. 2012-01-0744.

[31]D. Filev, J. Lu, K. Prakah-Asante and F. Tseng, (2009), Real-time driving behavior identification based on driver-in-the-loop vehicle dynamics and control, IEEE International Conference on Systems, Man and Cybernetics, pp. 2020-202.

[32]W. Wang, J. Xi and H. Chen, (2014), Modeling and Recognizing Driver Behavior Based on Driving Data: A Survey, Hindawi Publishing Corporation.

[33]C. Guardiola, D. Blance-Rodriguez and A. Reig, (2014), Modelling driving behaviour and its impact on the energy management problem in hybrid electric vehicles, International Journal of Computer Mathematics, Vol. 91, No. 1, pp. 147-156.

[34]A. Doshi and M. Trivedi, (2010), Examining the impact of driving style on the predictability and responsiveness of the driver: Real-world and simulator analysis, IEEE Intelligent Vehicles Symposium, pp. 232-237.

[35] Bolovinou, A., Bellotti, F., Amditis, A. and Tarkiani, M., (2014), Driving style recognition for co-operative driving: a survey, ADAPTIVE 2014
The Sixth Inrernational Conferende on Adaptive and Self-Adaptive Systems and Applicationa, pp. 73-78.

[36]Huang, X., Tan, Y., and He, X., (2011), An intelligent multi-feature statistical approach for the discrimination of driving conditions of a hybrid electric vehicle, IEEE Transactions on Intelligent Transportation Systems, Vol. 12, No. 2, pp. $453-465$.

[37] Ishibashi, M., Okuwa, M., and Akamatsu, M., (2007), Indices for characterizing driving style and their relevance to car following behavior, Annual Conference SICE, pp. 1132 - 1137.

[38]Dörr, D., Grabengiesser, D. and Gauterin, F., (2014), Online driving style recognition using fuzzy logic, IEEE 17th International Conference on Intelligent Transportation Systems, pp. 1021-1026.

[39] Gilman, E., Keskinarkaus, A., Tamminen, S., Pirttikangas, S., Röning, J., and Riekki, J., (2015), Personalised assistance for fuel-efficient driving, Transportation Research Part C: Emerging Technologies, Vol. 58, pp. 681-705.

[40] McCall, J. C., and Trivedi, M. M., (2007), Driver behavior and situation aware brake assistance for intelligent vehicles, Proceedings of the IEEE, Vol. 95, No. 2, pp. $374-387$.

[41]Xia, H., Boriboonsomsin, K., and Barth, M., (2013), Dynamic ecodriving for signalized arterial corridors and its indirect network-wide energy/emissions benefits, Journal of Intelligent Transportation Systems, Vol. 17, No. 1, pp. $31-41$

[42]M. Van Ly, S. Martin and M. Trivedi, (2013), Driver classification and driving style recognition using inertial sensors, Intelligent Vehicles Symposium (IV), IEEE, pp. 1040-1045, 2013.

[43]V. Vaitkus, P. Lengvenis and G. Zylius, (2014), "Driving style classification using long-term accelerometer information, 19th IEEE International Conference On Methods and Models in Automation and Robotics, pp. 641-644.

[44] Xiong, H., and Boyle, L. N., (2012), Drivers' adaptation to adaptive cruise control: Examination of automatic and manual braking, IEEE transactions on intelligent transportation systems, Vol. 13, No. 3, pp. 1468-1473.

[45]L. Xu, J. Hu, H. Jiang and W. Meng, (2012), Establishing style-orientated driver models by imitating human driving behaviors, IEEE Transactions on Intelligent Transportation Systems, Vol. 16, No. 5, pp. 2522-2530.

[46]Lenaers, G., (September, 2009), Real Life $\mathrm{CO}_{2}$ emission and consumption of four car powertrain technologies related to driving behaviour and road type, SAE Technical paper, No. 2009-24-0127, $9^{\text {th }}$ International Conference on Enigne and Vehicles, pp. 1-15.

[47] Syed, F. U., Filev, D., and Ying, H, (2007), Fuzzy rule-based driver advisory system for fuel economy improvement in a hybrid electric vehicle, Annual Meeting of the North American, pp. $178-183$.

[48] J. Neubauer and E. Wood, (2013), Accounting for the variation of driver aggression in the simulation of conventional and advanced vehicles, SAE Technical Paper, No. 2013-01-1453.

[49]J. Huang, Y.-K. Chin and W. Lin, (2012), Adaptive vehicle control system with driving style recognition, Washington, DC: U.S. Patent and Trademark Office Patent U.S. Patent No. 8,260,515.

[50]G. Kurz, A. Müller, T. Röhring-Gericke, R. Schöb, H. Tröster and A. Yap, (2012), Method and device for classifying the driving style of a driver in a motor vehicle. Washington, DC: U.S. Patent and Trademark Office Patent U.S. Patent No. 6,449,572.

[51]A. Augustynowicz, (2008), Preliminary classification of driving style with objective rank method, International journal of automotive technology, Vol. 10, No. 5, pp. 607-610.

[52]Won, J.-S., (May, 2003), Intelligent energy management agent for a parallel hybrid vehicle, Submitted to the Office of Graduate Studies of Texas A\&M University in partial fulfilment of the requirements for the degree of DOCTOR OF PHILOSOPHY, Pusan National University, Korea.

[53] Kim, J., Sim, H., \& Oh, J., (2012), The flexible EV/HEV and SOC band control corresponding to driving mode, driver's driving style and environmental circumstances, SAE Technical Paper.

[54]Ching, W.K., Huang, X., Ng, M.K., and Siu, T.K., (2013), Markov Chains. Models, Algorithms and Applications, International Series in Operations Research \& Management Science, Vol. 189, Springer Science + Business Media New York, Second Edition.

[55]Pentland, A., and Liu, A., (1999), Modeling and prediction of human Behaviour, Neural computation, Vol. 11, No. 1, pp. 229-242.

[56] Nechyba, M.C. and Xu, Y., (1996), On the fidelity of human skill models, IEEE International Conference in Robotics and Automation, Vol. 3, pp. 2688-2693. 
[57]Syed, F. U., Filev, D., and Ying, H., (2008), Real time advisory system for fuel economy improvement in a hybrid electric vehicle, Annual Meeting of the North American, pp. 1-6.

[58] Syed, F. U., Filev, D., Tseng, F., and Ying, H., (2009), Adaptive real-time advisory system for fuel economy improvement in a hybrid electric vehicle, Annual Meeting of the North American, pp. 1-7.

[59]Reichart, G., Friedmann, S., Dorrer, C., Rieker, H., Drechsel, E., and Wermuth, G. (September, 1998), Potentials of BMW driver assistance to improve fuel economy, in FISITA World Automotive Congress, Paris, Vol. 27, pp. 1-16.

[60]Rossetti, R. J., Almeida, J. E., Kokkinogenis, Z., and Gonçalves, J., (2013), Playing transportation seriously: Applications of serious games to artificial transportation systems, IEEE Intelligent Systems, No. 4, pp. 104-112

[61]Rass, S., Fuchs, S., and Kyamakya, K. (2008), A game-theoretic approach to co-operative context-aware driving with partially random behavior, Smart Sensing and Context, Lecture Notes in Computer Science Volume 5279, pp. 154-167.

[62]Fotouhi, A., Yusof, R., Rahmani, R., Mekhilef, S., \& Shateri, N., (2014), A review on the applications of driving data and traffic information for vehicles ' energy conservation, Renewable and Sustainable Energy Reviews, Vol. 37, pp. 822-833.

[63]C. Urmson, D. Dolgov and P. Nemec, (2014), "Driving pattern recognition and safety control,". Washington, DC: U.S. Patent and Trademark Office Patent U.S. Patent No. 8,634,980.

[64]Lin, C.-C., Jeon, S. Peng, H., and Lee, J.M., (2004), Driver pattern recognition for control of hybrid electric truck, Vehicle System Dynamics: International Journal of Vehicle Mechanics and Mobility, Vol. 42, No. 1-2, pp. 41-58.

[65] Yu, H., Tseng, F., \& McGee, R., (2012), Driving pattern identification for EV range estimation, IEEE International Electric Vehicle Conference, pp. 1-7.

[66]Zhang, S., and Xiong, R., (2015), Adaptive energy management of a PHEV based on driving pattern recognition and DP, Applied Energy, Vol. 155 , pp. 68-78.

[67]Bingham, C., Walsh, C., \& Carroll, S., (2012), Impact of driving characteristics on electric vehicle energy consumption and range, IET Intelligent Transport Systems, Vol. 6, No. 1, pp. 29-35.

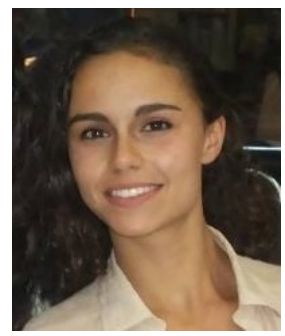

Clara Marina Martinez is a $\mathrm{PhD}$ student at Center for Automotive Engineering, Cranfield University, UK. She obtained the MSc degree in automotive mechatronics from Cranfield University in 2014. Her current research focuses on intelligent vehicle controls for electrified vehicles, driving style recognition and machine learning.

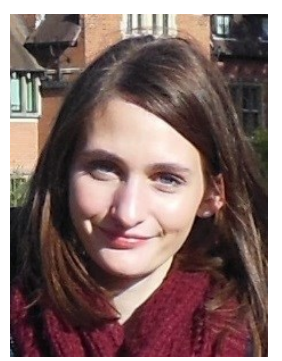

Mira Heucke received the M.Sc. degree in Automotive Mechatronics from Cranfield University U.K. in 2015 . She is currently taking part of a Trainee Junior Managers Program as part of Bosch GmbH in Leibniz Universität Hannover.

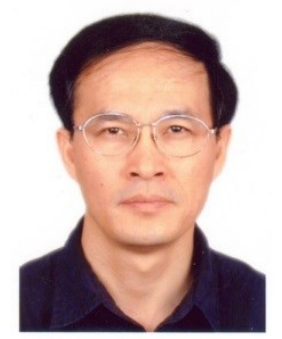

Fei-Yue Wang (S'87-M'89-SM'94-F'03) received the Ph.D. degree in computer and systems engineering from Rensselaer Polytechnic Institute, Troy, NY, in 1990. He is currently a Professor and the Director of the State Key Laboratory of Intelligent Control and Management of Complex Systems, Institute of Automation, Chinese Academy of Sciences, Beijing, China. Dr. Wang was the Founding Editor-in-Chief of the International Journal of Intelligent Control and Systems from 1995 to 2000 , the Series on
Intelligent Control and Intelligent Automation from 1996 to 2004, and IEEE INTELLIGENT TRANSPORTATION SYSTEMS. He was the Editor-inChief of IEEE INTELLIGENT SYSTEMS from 2009 to 2011 and is the Editor-in-Chief of IEEE TRANSACTIONS ON INTELLIGENT TRANSPORTATION SYSTEMS and IEEE/CAA JOURNAL OF AUTOMATICA SINICA. He was the President of the IEEE Intelligent Transportation Systems Society from 2005 to 2007, the Chinese Association for Science and Technology (CAST, USA) in 2005, and the American Zhu Kezhen Education Foundation from 2007 to 2008. Currently, he is the Vice President and Secretary General of the Chinese Association of Automation. $\mathrm{He}$ is a member of Sigma $\mathrm{Xi}$ and an elected fellow of INCOSE, IFAC, ASME, and AAAS

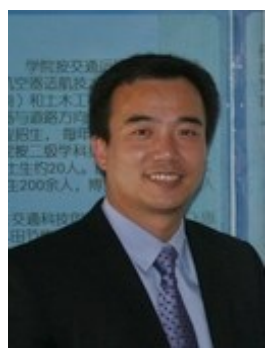

Bo Gao received the Ph.D. degree in Mechanical Engineering from University of Bath in UK. He is the department manager for Electronics \& Controls division in AVL Powertrain UK Ltd. He is now leading the vehicle electrification and ADAS activities within the company. $\mathrm{He}$ is also a member of AVL global controls committee. Since joining AVL in 2006 Dr. Gao has been working on various vehicle programs involved with several OEMs and Tier 1 suppliers. Prior to joining AVL, Dr. Gao was a research officer in the department of Mechanical Engineering in University of Bath, where he worked on vehicle dynamics and stability control. His PhD research was on the development of a novel hydro-pneumatic active suspension system supported by Jaguar Land Rover and Ford Motor Company. Dr Gao is a Chartered Engineer and a member of Institution of Mechanical Engineers. He holds one patent and has co-authored more than 20 technical papers

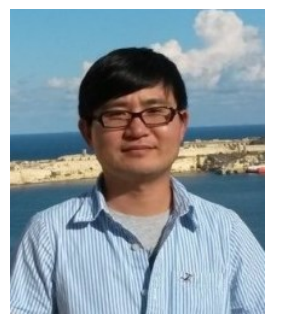

Dongpu Cao received the Ph.D. degree from Concordia University, Canada, in 2008. He is currently a Lecturer at Center for Automotive Engineering, Cranfield University, UK. His research focuses on vehicle dynamics, control and intelligence, where he has contributed more than 80 publications and 1 US patent. He received the ASME AVTT'2010 Best Paper Award and 2012 SAE Arch T. Colwell Merit Award. Dr. Cao serves as an Associate Editor for IEEE TRANSACTIONS ON INTELLIGENT TRANSPORTATION SYSTEMS, IEEE TRANSACTIONS ON VEHICULAR TECHNOLOGY and IEEE TRANSACTIONS ON INDUSTRIAL ELECTRONICS. He was a Guest Editor for VEHICLE SYSTEM DYNAMICS and IEEE/ASME TRANSACTIONS ON MECHATRONICS. He serves on the SAE International Vehicle Dynamics Standards Committee and a few ASME, SAE, IEEE technical committees. 
2017-07-04

\title{
Driving style recognition for intelligent vehicle control and advanced driver assistance: a survey
}

\author{
Marina Martinez, Clara
}

IEEE

Clara Marina Martinez, Mira Heucke, Fei-Yue Wang, Bo Gao and Dongpu Cao. Driving style recognition for intelligent vehicle control and advanced driver assistance: a survey. IEEE

Transactions on Intelligent Transportation Systems, Volume 19, Issue 3, March 2018, pp666-676

10.1109/TITS.2017.2706978

Downloaded from Cranfield Library Services E-Repository 